\title{
Increased bone metabolism in rheumatoid arthritis as measured by the whole-body retention of ${ }^{99} \mathrm{Tc}^{\mathrm{m}}$ methylene diphosphonate
}

\author{
C. RAJAPAKSE, R. THOMPSON, D. M. GRENNAN, B. M. WINSTON, \\ P. PATEL, P. M. NUTTALL, J. MURPHY, AND J. B. WEISS
}

From the University of Manchester, Rheumatic Diseases Centre, Hope Hospital, Salford; the Department of Rheumatology, South Manchester Teaching Hospital, Withington Hospital, Manchester; and the Regional Department of Medical Physics and Bioengineering, Christie Hospital, Manchester

SUMMARY Bone metabolism in 21 patients with rheumatoid disease was investigated by measurement of the 24-hour whole body retention (WBR) of ${ }^{99} \mathrm{Tc}^{\mathrm{m}}$ methylene diphosphonate (MDP) in parallel with clinical, radiological, and biochemical measurements (urinary excretion of hydroxyproline) of disease activity. Corticosteroid-treated patients of those with other forms of metabolic bone disease were excluded from the study. WBR was increased in the rheumatoid patients as compared with 21 age- and sex-matched controls $(p<0.05)$, and there was a significant correlation in the rheumatoid group between WBR and urinary excretion of hydroxyproline $(\mathrm{p}<0.01)$ and between urinary excretion of hydroxyproline and an articular index $(p<0.05)$ and global index $(p<0.01)$ of disease activity. The increased WBR of the rheumatoid patients was not explicable by factors such as immobilisation, and the results are interpreted as reflecting an overall increase in bone metabolism which may occur in rheumatoid arthritis as part of the disease process.

Rheumatoid arthritis is a chronic inflammatory disease associated in severe cases with progressive joint destruction and erosion of periarticular bone. There may be generalised as well as localised bone loss in rheumatoid patients, including those not treated with corticosteroids. ${ }^{12}$ The study of bone turnover in rheumatoid patients in parallel with clinical and biochemical measurements of disease activity would therefore seem relevant to the fuller understanding of this osteoporosis and could conceivably help in the serial assessment of disease activity. Radioisotope methods of imaging and probe counting have some value in the measurement of local bone activity but have limitations in terms of radiation dosage and of sensitivity. ${ }^{3}$ More recently the measurement of whole body retention (WBR) of a much smaller dose of ${ }^{99} \mathrm{Tc}^{\mathrm{m}}$ methylene diphosphonate (MDP) has been shown to detect increased bone metabolism in various forms of metabolic bone disease. ${ }^{4}$ The aim of the present study was to measure the 24-hour wholebody retention of MDP in patients with rheumatoid

Accepted for publication 12 March 1982.

Correspondence to Dr D. M. Grennan, Rheumatic Diseases Centre, Hope Hospital, Salford M6 8HD. arthritis of varying disease severity and in parallel with other clinical, radiological, and laboratory measurements of disease activity. The laboratory indices have included the measurement of urinary excretion of hydroxyproline, a marker of collagen breakdown and previously shown to correlate with disease activity in rheumatoid patients. ${ }^{5}$

\section{Patients and methods}

Twenty-one patients with classical or definite rheumatoid arthritis ${ }^{6}$ and aged from 31 to 74 years (mean 51 years) were examined. Six were outpatients and 15 were inpatients (mean time in hospital 6 days, range 1 to 19 days). Twenty-one age- and sexmatched controls who were either medical or laboratory personnel or patients with nonarticular rheumatic diseases were also studied.

Clinical disease activity in the rheumatoid patients was measured by an articular index ${ }^{7}$ and a global index of disease activity, scored on a $0-3$ basis. Hand and feet $x$-rays of the patients with rheumatoid arthritis were scored independently, with a maximum possible score of 40 in each patient. ${ }^{8}$ 


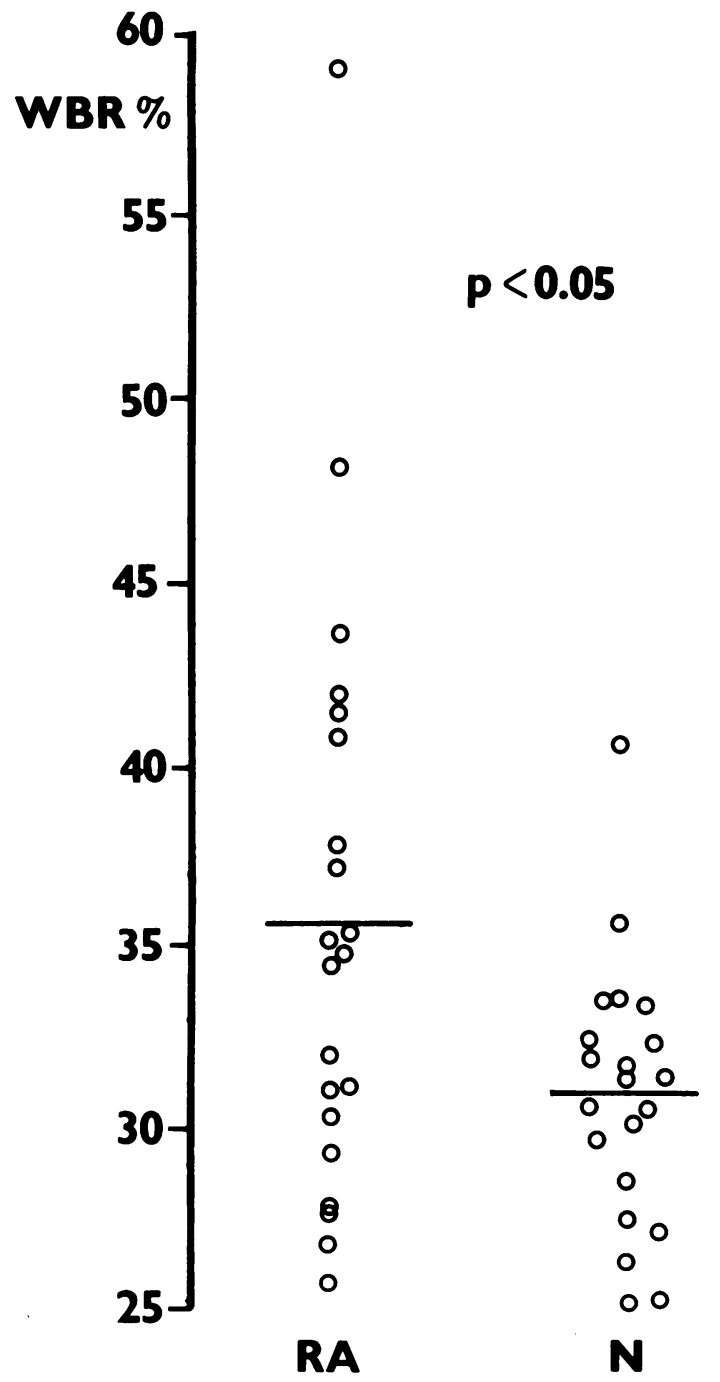

Fig. 1 WBR of MDP in rheumatoid $(R A)$ and control groups $(N)$.

Twenty-four-hour urinary excretion of hydroxyproline was measured with the Hypronosticon kit (Organon Teknika) in both the rheumatoid patients and the controls while they were receiving a gelatinfree diet. Results were expressed as $\mathrm{mg}$ per square metre surface area.

The whole body retention of MDP, 24 hours after intravenous injection of $0.4 \mathrm{MBq}$ was measured. Whole-body retention was measured with a shadow shield, whole-body monitor at 5 minutes and 24 hours after injection. Retention of the radiopharmaceutical was calculated ${ }^{4}$ as

$$
\frac{\text { net } 24 \text {-hour count }}{\text { net } 5 \text {-minute count }} \times 100
$$

The following were excluded from this study: patients receiving corticosteroids, patients with renal disease, and patients with other forms of metabolic bone disease detected by clinical and biochemical screening. Serum alkaline phosphatase levels were raised in 3 patients. In one this was accounted for by liver isoenzymes, and in the other 2 with alkaline phosphatase values of 101 units and 98 units respectively (normal range 20-95) their diets had been

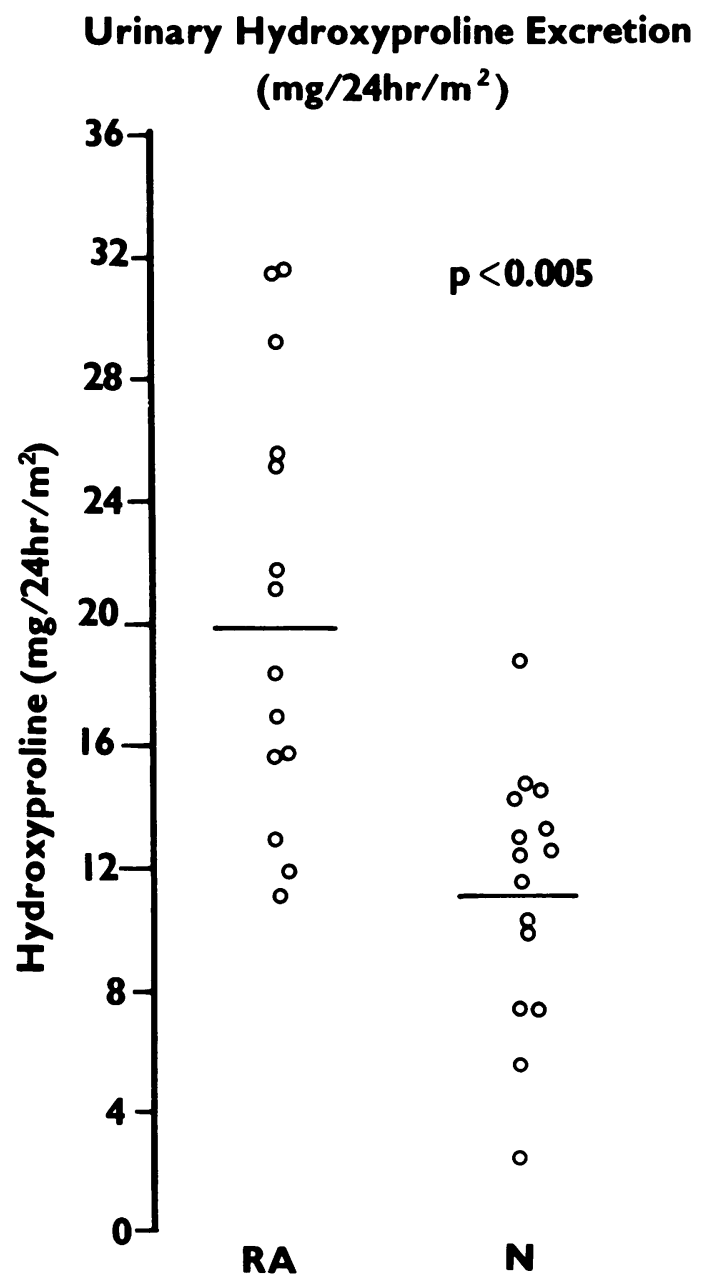

Fig. 2 24-hour urinary hydroxyproline in $R A$ and control groups. 


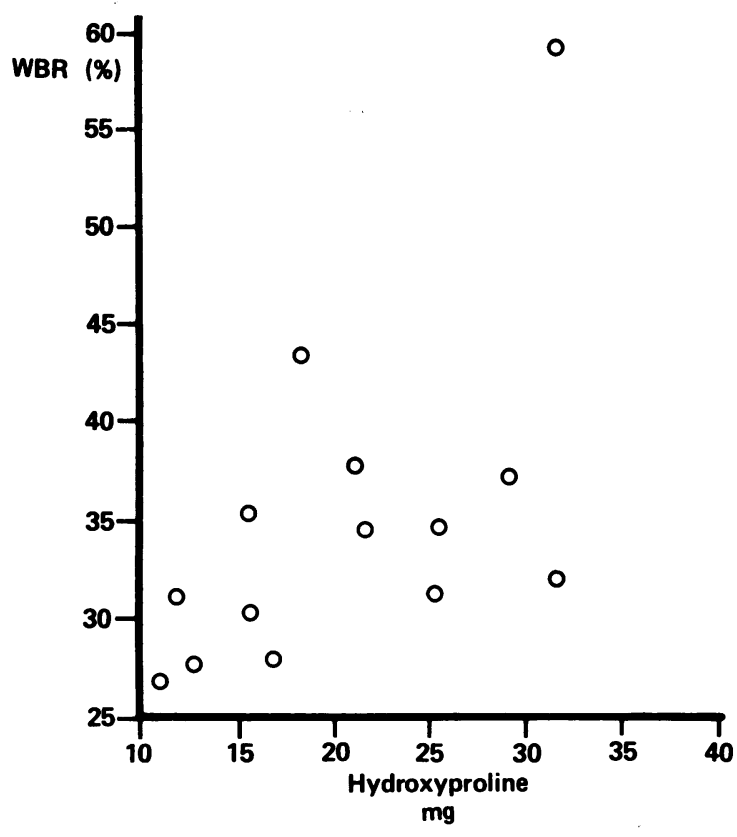

Fig. 3 Scatter diagram of relationship between WBR and urinary hydroxyproline in rheumatoid group.

adequate and radiological screen showed no evidence of other metabolic bone disease.

Differences in whole body retention of MDP between rheumatoid and control groups were analysed by the Mann-Whitney U test. ${ }^{9}$ Correlation coefficients were analysed by the nonparametric Spearman's test.

\section{Results}

The values for WBR and urinary excretion of hydroxyproline in the rheumatoid group were significantly higher than control values and are shown in Figs. 1 and 2 . There was a statistically significant correlation between hydroxyproline excretion and WBR in the rheumatoid group $(\mathrm{p}<0.01, \mathrm{Fig} .3)$. The values of both WBR and urinary excretion of hydroxyproline for outpatients and inpatients in the rheumatoid group similar. There was no significant correlation between WBR and articular index, global index of disease activity, $x$-ray grading, ESR, or disease duration. There was a significant correlation between hydroxyproline excretion and both articular index $(p<0.05)$ and global index $(p<0.01)$ of disease activity but not between urinary hydroxyproline and $x$-ray grading, ESR, or disease duration.

\section{Discussion}

We have shown that the whole body retention of MDP is increased in the group of rheumatoid patients investigated and that this measurement was correlated with the urinary excretion of hydroxyproline, a measure of collagen breakdown. There is evidence to suggest that 24-hour whole-body retention of MDP reflects changes in bone metabolism. This evidence includes the high bone to soft tissue ratios found on bone scanning from 5 hours onwards following intravenous injection of MDP, experimental data in animals showing high concentration of disphosphonate in bone after intravenous injection, ${ }^{10}$ and increases in the 24-hour retention of MDP in various forms of metabolic bone disease in man. ${ }^{4}$ Although in normal bone most of the diphosphonate is taken up by bone-crystal nuclei, the affinity of the radiopharmaceutical for both immature collagen and crystal nuclei may be important when bone turnover is increased. ${ }^{112}$ In our rheumatoid patients the uptake of MDP was correlated with the urinary excretion of hydroxyproline, a marker of collagen breakdown, which would be in keeping with collagen affinity influencing isotope uptake in our patients. Urinary excretion of hydroxyproline has been shown previously to be raised in rheumatoid patients, and as $50 \%$ of the body's collagen is within bone it may also reflect bone matrix turnover. ${ }^{5}$ The finding that the whole-body retention of diphosphonate was increased in some of the rheumatoid patients could thus reasonably be interpreted as reflecting an increase in bone metabolism in those patients. It is likely that this increased bone metabolism is part of the rheumatoid disease process and is not explicable by the presence of other causes of metabolic bone disease such as Paget's disease or by previous corticosteroid therapy, as care was taken to exclude such patients from the study. Both inpatients and outpatients had evidence of increased bone metabolism, so that the results were not explicable by factors associated with immobilisation or admission to hospital.

The increased whole-body retention of MDP could conceivably reflect a localised increase but seems more likely to represent a generalised increase in bone metabolism. The present findings are in keeping with those of a previous study, which showed an increased uptake of ${ }^{99} \mathrm{Tc}^{\mathrm{m}}$ pyrophosphate by nonjoint bone in rheumatoid patients and increased whole body retention of MDP in a smaller group of patients. ${ }^{13}$ There is histological as well as radiological evidence that generalised as well as localised bone loss may occur in rheumatoid patients not treated with corticosteroids. ${ }^{12}$ Furthermore Kennedy and coworkers ${ }^{14}$ have demonstrated bone 
resorbing activity in the serum of rheumatoid patients. Measurement of both local and general bone metabolism may thus be relevant to the serial assessment of the effects of the disease on bone. In the small number of patients we have so far investigated in this cross-sectional study the WBR measurements were correlated neither with radiological nor with clinical measurements of disease severity. This may be explained by the fact that the $x$-ray index reflects previous and not necessarily current bone destruction, while the clinical measurements may have been influenced by the nonsteroidal anti-inflammatory drugs which most of the patients were receiving. There was, however, a significant relationship between urinary hydroxyproline and both the articular index and the global index of disease activity, which is in keeping with previous suggestions that urinary hydroxyproline may be a useful objective measurement of rheumatoid disease activity. ${ }^{5}$ Further longitudinal studies are in progress to examine the usefulness of both hydroxyproline and isotope measurements in the assessment of active bone destruction in rheumatoid patients.

\section{References}

1 Duncan H, Frost H M, Villanueva A R, Sigler J W. The osteoporosis of rheumatoid arthritis. Arthritis Rheum 1965; 8: 943-54.

2 Kennedy A C, Smith D A, Anton H C, Buchanan W W. Generalised and localised bone loss in patients with rheumatoid arthritis. Scand J Rheumatol 1975; 4: 209-15.
3 Dick W C, Grennan D M. Radioisotopes in the study of normal and inflamed joints. Clin Rheum Dis 1976; 2: 67-76.

4 Fogelman I, Bessent R G, Turner J G, Citrin D L, Boyle I T, Greig W R. The use of whole-body retention of TC- $99 \mathrm{~m}$ diphosphonate in the diagnosis of the metabolic bone disease. $J$ Nucl Med Allied Sci 1977; 19: 170-275.

5 Manicourt D, Orloff S, Rao V H. Synovial fluid and serum hydroxyproline fraction in rheumatoid arthritis. Scand $J$ Rheumatol 1979; 8: 161-7.

6 Ropes M W, Bennet G A, Cobb S, Jacox R, Jessar R A. Revision of diagnostic criteria for rheumatoid arthritis. Arthritis Rheum 1958; 2: 16-20.

7 Ritchie D M, Boyle J A, McInnes J M, et al. Clinical studies with an articular index for the assessment of joint tenderness in patients with rheumatoid arthritis. $Q J$ Med 1968; 37: 393-406.

8 Larsen A, Dale K, Eek M. Radiographic evaluation of rheumatoid arthritis and related conditions by standard reference films. Acta Radiol (Diagn) (Stockh) 1977; 18: 481-91.

9 Siegel S. Non Parametric Statistics for the Behavioural Sciences. New York: McGraw-Hill, 1956: 202.

10 Rohlin M, Nosslin B. Quantitative analysis of the distribution of ${ }^{90 m} \mathrm{Tc}$-labelled ethylene hydroxy diphosphonate in young rats. Eur J Nucl Med 1977; 2: 113-5.

11 Guillemart A, Besnard J-C, LePape A, Galy G, Fetissoff F. Skeletal uptake of pyrophosphate labeled with technetium-95m and technetium-96, as evaluated by autoradiography. $J$ Nucl Med Allied Sci 1978; 19: 895-9.

12 Kaye M, Silverton S, Rosenthall C. Technetium-99mpyrophosphate: studies in vivo and in vitro.J Nucl Med Allied Sci 1975; 16: 40-5.

13 Rosenspire K C, Kennedy A C, Steinbach J, Blau M, Green F A. Investigation of the metabolic activity of bone in rheumatoid arthritis. J Rheumatol 1980; 7: 469-73.

14 Kennedy A C, Lindsay R, Buchanan W W, Allam B F. Boneresorbing activity in the sera of patients with rheumatoid arthritis. Clin Sci Mol Med 1976; 51: 205-7. 